\title{
CONCERNING INTRINSIC TOPOLOGIES ON BOOLEAN ALGEBRAS AND CERTAIN BICOMPACTLY GENERATED LATTICES
}

\author{
by C. R. ATHERTON
}

(Received 14 November, 1968; revised 30 September, 1969)

Introduction. This paper may be regarded as a continuation of the investigations begun in [2]; certain intrinsic lattice topologies are studied, especially the order and ideal topologies in Boolean algebras, bicompactly generated lattices, and other more general structures. The results of [1], [2], and [3] are shown to be closely related. It is proved that the ideal topology on any Boolean algebra has a closed subbase consisting of all sublattices, whereas the order topology on an atomic Boolean algebra has a closed subbase consisting of all sub-complete lattices. It is also shown that the order topology on an atomic Boolean algebra is autouniformizable (in the sense defined by Rema [3]) and, if the ground set is infinite, strictly coarser than the ideal topology. The conditions $\mathrm{Cl}$ and $\mathrm{C} 3$ on a lattice, introduced by Kent [1], are shown to be slightly stronger than the condition " bicompactly generated", and in complete lattices, where these conditions are satisfied, the order topology is shown to be coarser than the ideal topology.

\section{The ideal topology on a Boolean algebra.}

Definition. The ideal topology on a lattice $L$ is the topology on $L$ having an open subbase, consisting of the collection of all completely irreducible ideals and completely irreducible dual ideals of $L$. In this section it will be proved that on a Boolean algebra $B$ the ideal topology has a closed subbase consisting of all ideals and dual ideals of $B$. The proof begins with a lemma that makes use of the fact that on a Boolean algebra $B$ the following conditions concerning an ideal $I$ of $B$ are equivalent:

(i) $l$ is maximal.

(ii) $l$ is completely irreducible.

(iii) $I$ is maximal subject to not containing some element of $B$.

(iv) $B-I$ is a maximal dual ideal of $B$.

The notation $x^{*}=\{y \in L: y \geqq x\}$ and $x^{+}=\{y \in L: y \leqq x\}$, where $L$ is a lattice, is also used.

Lemma 1.1. Let $B$ be a Boolean algebra and $A \subset B$. Denote by $c(A)$ the closure of $A$ under the ideal topology on $B$. If $A$ is closed under finite joins then $x$ is in $c(A)$ if and only if $x$ is in $c\left(A \cap x^{*}\right)$; moreover, $x$ is in $c\left(A \cap x^{*}\right)$ only if $x$ is a finite meet of elements from $A \cap x^{*}$. Dually, if $A$ is closed under finite meets then $x$ is in $c(A)$ if and only if $x$ is in $c\left(A \cap x^{+}\right) ; x$ is in $c\left(A \cap x^{+}\right)$ only if $x$ is a finite join of elements from $A \cap x^{+}$.

Proof. Since $A \cap x^{*} \subset A$ it is clear that $c\left(A \cap x^{*}\right) \subset c(A)$. Conversely, suppose $x$ is in $c(A)$ but not in $c\left(A \cap x^{*}\right)$. Then there exist maximal ideals $I_{1}, \ldots, I_{n}$ of $B$ such that $x$ is in

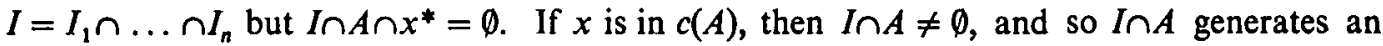


ideal $J^{\prime}$ of $B . \quad x$ is not in $J^{\prime}$, since $x$ is in $J^{\prime}$ if and only if $x$ is less than or equal to some finite join of elements from $\operatorname{In} A$; when $A$ is closed under finite joins this is equivalent to: $x$ is less than or equal to some element of $\operatorname{In} A$, in which case $\operatorname{In} A \cap x^{*}$ would be non-empty. Thus $x^{*} \cap J^{\prime}=\emptyset$, and so there exists a maximal ideal $J \supset J^{\prime}$, which is disjoint from $x^{*}$. Therefore $D=B-J$ is a maximal dual ideal of $B$ containing $x$. Consequently $D \cap I$ is an open set in the ideal topology, which contains $x$ and is disjoint from $A$, contradicting the assumption that $x$ is in $c(A)$. This proves that $x$ is in $c\left(A \cap x^{*}\right)$. If $x$ is not a finite meet of elements from $A \cap x^{*}$, then $A \cap x^{*}$ generates a dual ideal disjoint from $x^{+}$, from which it follows that $x$ is not in $c\left(A \cap x^{*}\right)$. Therefore, $x$ is in $c\left(A \cap x^{*}\right)$ only if $x$ is a finite meet of elements from $A \cap x^{*}$. The dual argument, in the case that $A$ is closed under finite meets, completes the proof.

Corollary 1.2. On a Boolean algebra $B$ the ideal topology has a closed subbase consisting of all sublattices of $B$.

Proof. It follows immediately from the lemma that every sublattice of $B$ is closed with respect to the ideal topology on $B$. Therefore the topology on $B$ having a closed subbase of all sublattices of $B$ is coarser than the ideal topology on $B$. Note that the ideal topology on $B$ has a closed subbase consisting of the collection of all completely irreducible ideals and dual ideals of $B$, and so to prove the corollary, it suffices to observe that every ideal and dual ideal of any lattice $L$ is a sublattice of $L$.

ThEOREM 1.3. On a Boolean algebra $B$, the ideal topology has a closed subbase consisting of all ideals and dual ideals of $B$.

Proof. The topology on $B$, having a closed subbase of all completely irreducible ideals and dual ideals, is coarser than the topology on $B$, having a closed subbase of all ideals and dual ideals of $\mathrm{B}$, which is in turn coarser than the topology on $B$, having a closed subbase of all sublattices of $B$. By the preceding corollary, the first and last of these topologies coincide with the ideal topology.

\section{The $T_{D}$ and $T_{I D}$-topologies on a Boolean algebra.}

A Boolean metric $d$ on the Boolean algebra $B$ is a mapping of $B \times B$ onto $B$ defined by $d(a, b)=a+b=\left(a \wedge b^{\prime}\right) \vee\left(a^{\prime} \wedge b\right)$, for any $a, b$ in $B$. The Boolean algebra $B$ together with the metric $d$ is an auto-metrized Boolean algebra. Rema [3] showed that, if $D$ is any dual ideal of $B$, then the sets $U_{p}=\{(x, y): d(x, y) \leqq p ; p \in D\}$ form a base for a uniformity which induces a uniform structure on $B$; the resulting topology $T_{D}$ is called an auto-topology on $B$. $A$ Boolean algebra $B$, together with an auto-topology $T_{D}$, is an auto-topologized Boolean algebra.

Kent and Atherton [2] have exhibited a family of topologies on a lattice $L$, defined in terms of ideals and dual ideals of $L$. Specifically, let $I$ and $D$ be respectively an ideal and a dual ideal of $L$. The $T_{I D^{-}}$topology on $L$ is that topology which has an open subbase consisting of all sets of the form $a^{*} \cap b^{+}$, where $a \in I$ and $b \in D$. The following proposition shows that on a Boolean algebra every auto-topology is a $T_{I D}$-topology.

THEOREM 2.1. Let $B$ be a Boolean algebra and $I$ an ideal of $B$. If $D$ is the dual ideal of $B$ consisting of all complements of elements in $I$, then $T_{I D}=T_{D}$ and is therefore auto-uniformizable. 
Proof. A $T_{D}$ neighborhood base about an element $a$ of $B$ consists of all sets of the form $A_{p}(a)=\{x \in B: d(a, x) \leqq p ; p \in D\}$, while a $T_{I D}$ neighborhood base about $a$ consists of all sets of the form $V_{c p}(a)=\{x \in B: a \wedge c \leqq x \leqq a \vee p$, where $c \in I$ and $p \in D\}$. Note that $V_{c p}(a)$ contains a symmetric $T_{I D}$ neighborhood $V_{b b^{\prime}}(a)$ of $a$, where $b=c \vee p^{\prime}$ and $b^{\prime}=c^{\prime} \wedge p$. Thus each $T_{I D}$ neighborhood filter has a base of symmetric neighborhoods, and to prove the theorem it suffices to show that, if $c^{\prime} \in D$, then $V_{c c^{\prime}}(a)=A_{c^{\prime}}(a)$. If $x$ is in $A_{c^{\prime}}(a)$, then from $\left(a \wedge x^{\prime}\right) \vee\left(a^{\prime} \wedge x\right) \leqq$ $c^{\prime}$ it follows that $a \wedge c \leqq x, x \leqq a \vee c^{\prime}$ and so $x$ is in $V_{c c^{\prime}}(a)$. Similarly, if $x$ is in $V_{c c^{\prime}}(a)$, then from $a \wedge c \leqq x \leqq a \vee c^{\prime}$ it can be shown that $a^{\prime} \wedge x \leqq c^{\prime}, a \wedge x^{\prime} \leqq c^{\prime}$, and so $x$ is in $A_{c^{\prime}}(a)$; this proves that $V_{c c^{\prime}}(a)=A_{c^{\prime}}(a)$ and therefore $T_{I D}=T_{D}$.

A well known representation theorem concerning lattices is that any distributive lattice is isomorphic to a ring of sets, and hence can be embedded in some Boolean algebra. If $L$ is a distributive lattice, consider $L$ as being embedded in the Boolean algebra $B$, and further assume that $I$ is an ideal of $L$ such that $a^{\prime}$ is in $L$ for every $a$ in $I$, complementation being taken in $B$. Then $\left\{a^{\prime}: a\right.$ is in $\left.I\right\}$ generates a dual ideal of $L$, which in turn generates a dual ideal $K$ of $B$. In terms of this notation a more general form of the preceding theorem can now be stated.

THEOREM 2.2. If $T_{K}$ denotes the auto-topology on $B$ determined by $K$ and $T$ is the topology induced on $L$ by $T_{K}$, then $T=T_{I D}$.

Proof. Let $J$ be the ideal of $B$ generated by $I$. By Theorem 2.1, $T_{J K}=T_{K}$ on $B$, and so $T$ coincides with the topology induced on $L$ by $T_{J K}$. It remains to prove that $T=T_{I D}$. A $T_{I D}$-neighborhood base about $a$, an element of $L$, consists of all sets of the form $V_{j k}(a)=$ $L \cap\{z \in B: a \wedge j \leqq z \leqq a \vee k$, where $j \in J$ and $k \in K\}$. If $c=j$ and $d=k$, then $V_{c d}(a)=V_{j k}(a)$ and the $T_{I D}$-neighborhood filter at $a$ is coarser than the $T$-neighborhood filter at $a$. If $j$ is in $J$ and $k$ is in $K$ then there exist $c$ in $I$ and $d$ in $D$ such that $c \leqq j$ and $d \leqq k$ so that $V_{c d}(a) \subset$ $V_{j k}(a)$. Therefore, the $T_{I D}$-neighborhood filter at $a$ is finer than the $T$-neighborhood filter at $a$, from which it follows that $T=T_{I D}$ and the proof is complete.

The order topology on a lattice is usually defined in terms of order convergence, the sets which are closed in the order topology being described in terms of filter convergence. However, in any bicompactly generated lattice the order topology can be described in terms of ideals and dual ideals. An element $c$ of a lattice $L$ is compact if $S \subset L$ and $c \leqq \sup S$ implies $c \leqq \sup F$ for some finite set $F \subset S$. The set of all compact elements of $L$, denoted by $C(L)$, is closed under finite joins. An element $d$ of a lattice $L$ is cocompact if $S \subset L$ and $d \geqq \inf S$ implies $d \geqq \inf F$ for some finite set $F \subset S$. The set of all cocompact elements of $L$, denoted by $C C(L)$, is closed under finite meets. A lattice $L$ is compactly generated if, for every $x$ in $L$, $x=\sup \left(x^{+} \cap C(L)\right)$, and is bicompactly generated if, in addition, $x=\inf \left(x^{*} \cap C C(L)\right)$. Kent and Atherton [2] showed that on a bicompactly generated lattice $L$ the order topology is Hausdorff, totally disconnected, and coincides with $T_{C}$; also $T_{C}=T_{I D}$, where $I$ is the ideal of $L$ generated by $C(L)$ and $D$ is the dual ideal of $L$ generated by $C C(L)$. A corollary of this result follows.

THEOREM 2.3. The order topology on an atomic Boolean algebra $B$ is B-metrizable.

Proof. Let $D$ be the dual ideal of $B$ generated by the coatoms of $B ; D$ coincides with 
the dual ideal of $B$ generated by $C C(B)$. If $I=\left\{a^{\prime}: a \in D\right\}$, then $I$ is the ideal of $B$ generated by $C(B)$. The result mentioned above together with Theorem 2.1 yields the fact that the order topology coincides with $T_{C}=T_{I D}=T_{D}$, and $T_{D}$ is $B$-metrizable.

An analogue of Theorem 2.3 is obtained for the ideal topology, upon consideration of the Stone space of a Boolean algebra $B$. If $X$ is the Stone space of the Boolean algebra $B$, it can be shown that the order topology on $2^{X}$ induces the ideal topology on $B$. This fact together with the preceding theorem yields the following result.

THEOREM 2.4. The ideal topology on the Boolean algebra $B$ is induced by the B-metric on $2^{X}$, where $X$ is the Stone space of $B$.

\section{Conditions related to the concept of bicompactly generated lattices.}

As mentioned above, the order topology can be described in terms of ideals and dual ideals in any bicompactly generated lattice. Kent [1] has given conditions under which the order topology has an open subbase consisting of a certain family of ideals and dual ideals. Let $L(x)$ denote the intersection of all ideals $I$ of the lattice $L$ such that $\sup I=x$, and $U(x)$ the intersection of all dual ideals $D$ of $L$ having inf $D=x$. Kent's three conditions are:

Cl: $x=\sup L(x)=\inf U(x)$ for all $x$ in $L$.

C2: If $x \in L(y)$, then $U(x) \cap L(y) \neq \emptyset$. If $x \in U(y)$, then $L(x) \cap U(y) \neq \emptyset$.

C3: If $x \in L(y)$ and $x \leqq z$, then $x \in L(z)$. If $x \in U(y)$ and $x \geqq z$, then $x \in U(z)$.

He then showed that, if a lattice $L$ satisfies $\mathrm{C} 1$ and $\mathrm{C} 2$, the order topology has an open subbase consisting of all ideals $L(x)$ and dual ideals $U(x)$, where $x$ is in $L$, and if $L$ satisfies $C 1, \mathrm{C} 2$, and $\mathrm{C} 3$, then the order topology is discrete. Moreover, in a lattice satisfying $\mathrm{Cl}$ and either $\mathrm{C} 2$ or C3, the neighborhood filter about $x$ in the order topology has a base consisting of sets of the form $a^{*} \cap b^{+}$, where $a$ is in $L(x)$ and $b$ is in $U(x)$, for any $x$ in the lattice.

That the conditions $\mathrm{C} 1, \mathrm{C} 2$, and $\mathrm{C} 3$ are closely related to the notion of bicompactly generated lattices is shown by the next two theorems.

THEOREM 3.1. A lattice $L$ satisfies $\mathrm{Cl}$ and $\mathrm{C} 3$ if and only if $L$ is bicompactly generated, $C(L)$ is an ideal, and $C C(L)$ is a dual ideal.

Proof. Assume that $L$ satisfies $\mathrm{Cl}$ and C3. If $a$ is in $L(x)$ and $a \leqq \sup S=b$ for some set $S \subset L$, then $a$ is in $L(b)$. Letting $I$ be the ideal of $L$ generated by $S$ yields $\sup I=b$ and so $I \supset L(b)$. Therefore $a$ is in $I$, which implies that $a \leqq s_{1} \vee \ldots \vee s_{n}$ for some finite collection of $s_{i}$ from $S$; that is, $a$ is compact. Thus every element of $L(x)$ is compact, and $L$ is compactly generated. The dual argument proves that $L$ is bicompactly generated. The set $C(L)$ is closed under finite joins. Therefore, to prove $C(L)$ an ideal it remains to show that, if $x$ is less than a compact element of $L$, then $x$ is compact. Suppose $x<c$ and $c$ is compact; then $\sup L(c)=c$ implies $L(c)=c^{+}$. Since every element of $L(z)$ is compact for any $z$ in $L$, $x$ in $L(c)$ proves that $x$ is compact. The dual argument proves that $C C(L)$ is a dual ideal. 
Conversely, if $L$ is bicompactly generated, $C(L)$ is an ideal, and $C C(L)$ is a dual ideal, then assume that $c$ is in $x^{+} \cap C(L)$. If $I$ is an ideal of $L$ such that $\sup I=x \geqq c$, then, by compactness of $c, c$ is in $I$. Thus $x^{+} \supset L(x) \supset x^{+} \cap C(L)$, and $x=\sup x^{+} \geqq \sup L(x) \geqq \sup \left(x^{+} \cap C(L)\right)=x$. Similarly, $x=\inf U(x)$ and so $C l$ is satisfied. Since $\sup \left(x^{+} \cap C(L)\right)=x$ and $x^{+} \cap C(L)$ is an ideal, $L(x)=x^{+} \cap C(L)$. Thus, if $x$ is in $L(y)$ and $x \leqq z$, then, since $x$ is in $C(L), x$ must be in $z^{+} \cap C(L)=L(z)$. The dual argument proves that $L$ satisfies $C 3$, and the proof is complete.

THEOREM 3.2. In a lattice $L$ satisfying $\mathrm{C} 1$ and $\mathrm{C} 3$, condition $\mathrm{C} 2$ holds if and only if $L=C(L)=C C(L)$.

Proof. If $L$ satisfies $\mathrm{C} 1, \mathrm{C} 2$, and $\mathrm{C} 3$, then $x$ compact implies $x^{+}=L(x)$, in which case $U(x)=x^{*}$ and so $x$ must be compact. Similarly, every cocompact element of $L$ is compact, i.e., $C(L)=C C(L) \subset L$. If $x$ is in $L$, then $x=\sup \left(x^{+} \cap C(L)\right)$ implies that $x$ is in the dual ideal generated by $C(L)=C C(L)$; hence $x$ is cocompact and $L=C C(L)=C(L)$. Conversely, if $L=C(L)=C C(L)$, then, by the preceding theorem, $L$ satisfies $C 1$ and C3. If $x$ is in $L(y)$, then $x^{*} \cap L(y)=U(x) \cap L(y)$ is non-empty. Similarly, if $y$ is in $U(x)$, then $L(y) \cap U(x)$ is non-empty and condition $\mathrm{C} 2$ is satisfied.

Ward [5] has given an example to show that the ideal and order topologies may not be comparable on a Boolean algebra. It is a corollary of the next theorem that, on infinite atomic Boolean algebras, the ideal topology is strictly finer than the order topology.

THEOREM 3.3. On any complete lattice satisfying $\mathrm{Cl}$ and $\mathrm{C} 3$ the ideal topology is finer than the order topology.

Proof. Every element of a complete bicompactly generated lattice can be represented as a meet of completely meet irreducible elements as well as by a join of completely join irreducible elements [4, pp. 66-7]. Therefore, if $b$ is cocompact, $b$ can be expressed as a finite meet of completely meet irreducible elements of $L$. Thus $b=b_{1} \wedge \ldots \wedge b_{n}$, where the $b_{i}$ are completely meet irreducible; in this case $b^{+}=b_{1}^{+} \cap \ldots \cap b_{n}^{+}$is open in the ideal topology, since each $b_{i}^{+}$ is a completely irreducible ideal. Dually, if $a$ is compact, then $a^{*}$ is open in the ideal topology. Since, in a bicompactly generated lattice, the order topology coincides with $T_{c}$, this proves the theorem.

COROLlary 3.4. On any infinite atomic Boolean algebra B, the ideal topology is strictly finer than the order topology.

Proof. Each cocompact element of $B$ may be expressed as a finite meet of coatoms; hence $b^{+}$is a finite intersection of completely irreducible ideals generated by coatoms. Dually, $a^{*}$ is open in the ideal topology for every compact element $a$ of $B$. Thus, the sets $a^{*} \cap b^{+}$ ( $a$ compact, $b$ cocompact) are open in the ideal topology; since they form an open subbase for the order topology on $B$, the ideal topology is finer than the order topology. Since the ideal $C(B)$, consisting of all compact elements of $B$, is closed under the ideal topology, by Theorem 1.3, but the closure of $C(B)$, under the order topology, is $B$, it follows that the ideal topology is strictly finer than the order topology on $B$. 
It is an open question as to whether or not the ideal topology is Hausdorff on every distributive lattice, but it follows immediately from Theorem 3.3 that on any complete distributive lattice, satisfying $\mathrm{Cl}$ and $\mathrm{C} 3$, the ideal topology is Hausdorff, since the order topology is Hausdorff on such lattices.

The results in this paper are from the author's dissertation written at the Washington State University. In this connection I wish to express my gratitude to Dr D. C. Kent for his guidance.

\section{REFERENCES}

1. D. C. Kent, On the order topology in a lattice, Illinois J. Math. 10 (1966), 90-96.

2. D. C. Kent and C. R. Atherton, The order topology in a bicompactly generated lattice $J$. Australian Math. Soc. 8 (1968), 345-349. 221-243.

3. P. S. Rema, Auto-topologies in Boolean algebras, J. Indian Math. Soc. (N.S.) 30 (1967),

4. G. Szasz, Introduction to Lattice Theory (New York, 1963).

5. A. J. Ward, On relations between certain intrinsic topologies in partially ordered sets, Proc. Cambridge Philos. Soc. 51 (1955), 254-261.

DalhousIE UNIVERSITY

Halifax, Canada 